\title{
Novel mutations in NSP-1 and PLPro of SARS-CoV-2 NIB-1 genome mount for effective therapeutics
}

\author{
Mohammad Uzzal Hossain ${ }^{1 \dagger}$, Arittra Bhattacharjee ${ }^{1,2+}$, Md. Tabassum Hossain Emon ${ }^{3 \dagger}$, \\ Zeshan Mahmud Chowdhury², Ishtiaque Ahammad', Md. Golam Mosaib4, Md. Moniruzzaman5,

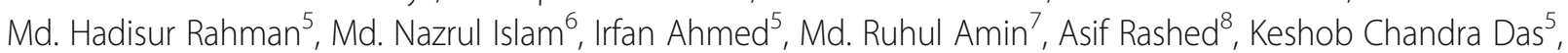 \\ Chaman Ara Keya ${ }^{2}$ and Md. Salimullah ${ }^{5^{*}}$ (D)
}

\begin{abstract}
Background: Severe acute respiratory syndrome coronavirus-2 (SARS-CoV-2), the etiologic agent of coronavirus disease 2019 (COVID-19), is rapidly acquiring new mutations. Analysis of these mutations is necessary for gaining knowledge regarding different aspects of therapeutic development. Previously, we have reported a Sanger methodbased genome sequence of a viral isolate named SARS-CoV-2 NIB-1, circulating in Bangladesh. The genome has four novel non-synonymous mutations in V121D, V843F, A889V, and G1691C positions.

Results: Using different computational tools, we have found V121D substitution has the potential to destabilize the non-structural protein-1 (NSP-1). NSP-1 inactivates the type-1 interferon-induced antiviral system. Hence, this mutant could be a basis of attenuated vaccines against SARS-CoV-2. V843F, A889V, and G1691C are all located in nonstructural protein-3 (NSP-3). G1691C can decrease the flexibility of the protein. V843F and A889V might change the binding pattern and efficacy of SARS-CoV-2 papain-like protease (PLPro) inhibitor GRL0617. V843F substitution in PLPro was the most prevalent mutation in the clinical samples. This mutation showed a reduced affinity for interferon-stimulated gene-15 protein (ISG-15) and might have an impact on innate immunity and viral spread. However, V843F+A889V double mutant exhibited the same binding affinity as wild type PLPro. A possible reason behind this phenomenon can be that V843F is a conserved residue of PLPro which damaged the protease structure, but A889V, a less conserved residue, presumably neutralized that damage.

Conclusions: Mutants of NSP-1 could provide attenuated vaccines against coronavirus. Also, these mutations of PLPro might be targeted to develop better anti-SARS therapeutics. We hope our study will help to get better insides during the development of attenuated vaccine and PLPro inhibitors.
\end{abstract}

Keywords: SARS-CoV-2, COVID-19, NSP-1, Papain-like protease, GRL0617, ISG-15

\footnotetext{
* Correspondence: salim2969@gmail.com

Mohammad Uzzal Hossain, Arittra Bhattacharjee, Md. Tabassum Hossain

Emon represent the first authors.

Mohammad Uzzal Hossain, Arittra Bhattacharjee, Md. Tabassum Hossain

Emon equally contributed to this manuscript.

${ }^{5}$ Molecular Biotechnology Division, National Institute of Biotechnology,

Ganakbari, Ashulia, Savar, Dhaka 1349, Bangladesh

Full list of author information is available at the end of the article
}

\section{Springer Open}

(c) The Author(s). 2021 Open Access This article is licensed under a Creative Commons Attribution 4.0 International License, which permits use, sharing, adaptation, distribution and reproduction in any medium or format, as long as you give appropriate credit to the original author(s) and the source, provide a link to the Creative Commons licence, and indicate if changes were made. The images or other third party material in this article are included in the article's Creative Commons licence, unless indicated otherwise in a credit line to the material. If material is not included in the article's Creative Commons licence and your intended use is not permitted by statutory regulation or exceeds the permitted use, you will need to obtain permission directly from the copyright holder. To view a copy of this licence, visit http://creativecommons.org/licenses/by/4.0/. 


\section{Background}

Severe acute respiratory syndrome coronavirus-2 (SARS$\mathrm{CoV}-2)$, a positive $(+)$ stranded RNA virus that initiated coronavirus disease 2019 (COVID-19) pandemic, is rapidly changing and adapting in different environments [1]. At the beginning of the COVID-19 outbreak, this viral infection was clinically manifested as flu-like symptoms (e.g., cough, fever, and trouble breathing) [2]. However, the acellular pathogen is frequently evolving with novel mutations, and in consequence, viral diarrhea, cardiovascular injury, strokes, psychosis, dementia or Kawasaki like syndrome are becoming more regular in COVID-19 cases [3-5]. According to the World Health Organization (WHO), this constant evolution is taking place due to different selection pressures that are letting the virus to be airborne and more resilient [6]. Proper analysis of these genomic mutations can help to decipher issues regarding viral transmission, epidemiology, pathogenicity, and therapeutics.

Coronaviruses (CoVs) are enveloped viruses that belong to the Coronaviridae family and Orthocoronavirinae subfamily $[7,8]$. Previously reported SARS-CoV-1 and Middle East respiratory syndrome coronavirus (MERS$\mathrm{CoV}$ ) belong to the Betacoronavirus genus of Orthocoronavirinae subfamily $[7,8]$. These pathogens were the causative agents of SARS and MERS epidemics. SARSCoV-2, a member of Betacoronavirus, shares nearly $82.30 \%$ sequence identity with SARS-CoV-1 while showing strikingly less similarity with MERS-CoV (28\%) [9].

The 29.9-kb RNA genome of SARS-CoV-2 has 11 genes with two untranslated regions (UTRs) at $5^{\prime}$ and $3^{\prime}$ terminals. Nearly $67 \%$ of the genome consists of Orf1ab polyprotein gene. The rest of the genome encodes structural proteins (e.g., surface (S), envelope (E), membrane $(\mathrm{M})$, and nucleocapsid (N) proteins) [10]. According to the Global initiative on sharing all influenza data (GISA ID) database, with particular prevalent mutations, the virus has made several distinct clades such as $\mathrm{S}, \mathrm{L}, \mathrm{V}, \mathrm{G}$, GR, GV, GRY and GH [11]. Among them, GR is one of the largest and predominates in South America, Europe, and Asia (http://www.gsaid.org). The members of this clade contain S-D614G and N-G204R mutations. Currently, GR is relatively less prevalent in North America. However, we have sequenced a GR-type viral isolate SARS-CoV-2/human/BGD/NIB_01/2020 (SARS-CoV-2 NIB-1) (NCBI GenBank Accession: MT509958, GISAID Accession ID: EPI_ISL_447904) from a young female COVID-19 patient from Bangladesh [12]. According to our report, the viral isolate shares a common ancestor with three North American SARS-CoV-2 isolates that were collected from Washington, United States of America (USA). Additionally, our isolate showed longer branch length or more genomic mutations compared with its closely related members [12]. When we aligned the genome with the reference genome (Accession: $\mathrm{NC}_{-}$ 045512.2), we found 11 mutations of which 4 of them were new. Amongst the new four mutations, three were found inside nonstructural protein-3 (NSP-3), of which two were located in the papain-like protease (PLPro) domain, and one was situated in the C-terminal domain of NSP-3. The remaining one was detected in nonstructural protein-1 (NSP-1) (Table 1).

NSP-1 and PLPro from SARS-CoV-1 or SARS-CoV-2 impede the type 1 interferon (IFN) antiviral activities of the host cells [13]. PLPro cleaves off interferonstimulating gene-15 (ISG-15) protein; therefore, the host cells cannot execute antiviral signals properly $[14,15]$. In this study, we have analyzed the effect of these novel mutations on the respective protein structures. We have shown that some of these mutations have the potential

Table 1 Mutations in SARS-COV-2 NIB-1

\begin{tabular}{|c|c|c|c|c|c|}
\hline SL no. & Reference position & Position in NIB-1 & Reference $\rightarrow$ NIB-1 & Change of amino acid & Coding region/ Gene \\
\hline \multicolumn{6}{|c|}{ Previously reported mutations } \\
\hline 1 & 241 & 93 & $\mathbf{C} \rightarrow \mathrm{T}$ & - & 5' UTR \\
\hline 2 & 1163 & 1015 & $\mathbf{A} \rightarrow T$ & I120F & Orf1ab \\
\hline 3 & 629 & 481 & $\mathrm{C} \rightarrow \mathrm{A}$ & L122I & Orf1ab \\
\hline 4 & 8171 & 8023 & $\mathbf{G} \rightarrow \mathrm{A}$ & $\mathrm{A} 1818 \mathrm{~T}$ & Orf1ab \\
\hline 5 & 23,403 & 23,255 & $A \rightarrow G$ & D614G* & S \\
\hline 6 & 28,882 & 28,734 & $\mathbf{G} \rightarrow A$ & R203K & N \\
\hline 7 & 28,883 & 28,735 & $\mathbf{G} \rightarrow C$ & G204R* & $\mathrm{N}$ \\
\hline \multicolumn{6}{|c|}{ Novel mutations } \\
\hline 8 & 627 & 479 & $\mathbf{T} \rightarrow \mathrm{A}$ & V121D & Orf1ab \\
\hline 9 & 5246 & 5098 & $\mathbf{G} \rightarrow \mathrm{T}$ & V843F & Orf1ab \\
\hline 10 & 5385 & 5237 & $\mathbf{C} \rightarrow \top$ & A889V & Orf1ab \\
\hline 11 & 7790 & 5642 & $\mathbf{G} \rightarrow T$ & G1691C & Orf1ab \\
\hline
\end{tabular}

Asterisk $\left(^{*}\right)$ containing D614G and G204R made the strain a member of the GR clade. 
to destabilize the structure of NSP-1 and the C-terminal domain of NSP-3. We also explored how the loss and gain of Valine in V843F and A889V mutations respectively affect the binding of protease inhibitors GRL0617 and ISG-15 for their implication in new therapeutics development.

\section{Methods}

\section{Genome retrieval and identification of the mutations}

The genome of SARS-CoV-2 NIB-1 was collected from the National Center for Biotechnology Information (NCBI) (GenBank accession: MT509958.1). The genome was aligned with the reference genome by NCBI Nucleotide Basic Local Alignment Search Tools (BLASTN) to identify the mutations in the Untranslated Regions (UTRs) [16]. The non-synonymous mutations were collected from GISAID CoVsurver (http://www.gisaid.org).

\section{Effect of the mutations on the proteins}

Mutations that affect the structural stability of the proteins can behave differently than the wild type one [17, 18]. To assess the effect of the novel mutations, MUpro, Protein Variation Effect Analyzer (PROVEAN), and HOPE were employed [19-21]. MUpro implemented Support Vector Machine and Neural Network to predict the results of the mutations. HOPE and PROVEAN used different programs to evaluate the possible outcomes due to the change of the amino acids in the proteins. Later, PLPro was taken for further analysis because of the availability of structural and molecular mechanisms on this protein.

\section{In silico mutagenesis, molecular modeling, and refinement of PLPro}

To observe the mutational effect of the protein at a threedimensional (3D) level, the crystal structure of PLPro was collected from Research Collaboratory for Structural Bioinformatics (RCSB) Protein Data Bank (PDB) (http://www. rcbs.org) [22]. The chain A of PLPro (PDB ID: 6W9C) was preserved via BIOVIA Discovery Studio (https://www.3ds. $\mathrm{com} /$ ). Heterogeneous atoms were also removed. Mutated protein sequences went under homology modeling by SWIS S-MODEL using chain A of 6W9C as a template [23]. The generated structures were energetically minimized by 3D Refine and Galaxy Refine [24, 25].

\section{Structural assessment of mutant proteins}

The structural quality of the mutant proteins was evaluated. To execute this step, RAMPAGE, ERRAT, VERI FY3D, PROVE, and SWISS-MODEL structure assessments were employed [23, 26-29].

\section{Inhibitor binding analysis against the wild type and} mutant PLPros

GRL0617 or 5-Amino-2-methyl-N-[(R)-1-(1-naphthyl) ethyl] benzamide (PubChem CID: 24941262) is a PLPro inhibitor with a relatively low level of cytotoxicity [30]. This naphthalene-based small molecule has the potential to be a prospective antiviral drug. Here, we analyzed the interactions between GRL0617 and PLPro by AutoDock tools 1.5.6 using our previously reported methodology for exploring drug-receptor interactions $[31,32]$. In short, the GRL0616 was taken as a ligand, while the wild type and mutant proteins were used as receptors. The active site of the palm domain in PLPro was enclosed with Grid Box [33]. The wild type PLPro (dimension points: $X=92, Y=74, Z=112$ ) was enclosed with $0.425 \AA$ spacing. A889V (dimension points: $X=60, Y=74, Z=96$ ) and V843F mutants (dimensions points: $X=64, Y=86, Z=102$ ) were enclosed with 0.531 and $0.499 \AA$ spacing, respectively. $\mathrm{V} 843+\mathrm{A} 889 \mathrm{~V}$ double mutant (dimension points: $X=$ 92, $Y=88, Z=112$ ) had $0.375 \AA$ spacing (detail parameters are given in Supplementary file 1). The molecular interactions between GRL0617-PLPro were visualized by UCSF Chimera [34], BIOVIA Discovery Studio and PyMOL Molecular Graphics System, Version 2.3.3 Schrödinger, LLC. Molecular dynamics (MD) simulations were carried out using Newtonian equations of motion via GROMACS 2020.1 [35]. Here, the PDB files of drug-receptor complexes were energetically minimized using GROMOS96 43a1 force field [36]. The protein topologies were built using pdb2gmx module within GROMACS, while the ligand topology was built using the PRODRG 2.5 server [37]. The protein-ligand complexes were solved in simple point charge (SPC) water model within a dodecahedron [38]. A minimum of 1 nanometer $(\mathrm{nm})$ distance was maintained between the protein surfaces and the edges of the box inside the unit cells. Each system was neutralized by adding 3 chlorine ions. Then the energy minimization was performed using the "grompp" module. The maximum force allowed was kept below $10 \mathrm{~kJ} / \mathrm{mol} / \mathrm{nm}$ tp. After restraining the ligand and setting up temperaturecoupling groups by combining the protein and the inhibitor, isothermal-isochoric (NVT) equilibration were done for 100 picoseconds (ps). Following the NVT equilibration, isothermal-isobaric (NPT) equilibration were carried out for the same duration. Finally, 50 nanoseconds (ns) of molecular dynamic simulation was carried out using $300 \mathrm{~K}$ temperature, 1 bar pressure, 1.2, short-range Van der Waals cutoff of $1.2 \mathrm{~nm}$, Particle Mesh Ewald for long-range electrostatics, and periodic boundary conditions. The trajectories of atoms were recorded every 2 femtoseconds (fs). 


\section{Analysis of ISG-15-PLPro interactions}

ISG-15 C-terminal domain that interacts with PLPro was collected from PDB ID: 6XA9. As a ligand/substrate, this protein was docked against the PLPro receptors by GalaxyTongDock_A [39]. After docking, Model 1 PDB files were taken since they had the highest cluster sizes and docking scores. The selected models were further analyzed by PROtein binDIng enerGY prediction (PRODIGY) to determine the binding affinity of the protein complexes at $25^{\circ} \mathrm{C}$ temperature [40].

\section{Detection of the novel mutations in clinical samples}

To identify the presence of these mutations in other SARS-CoV-2 isolates, we have collected 27 samples from July 2020 to November 2020 with their clinical data. All of the patients were tested positive for COVID-19 via Reverse transcription-polymerase chain reaction (RTPCR). The SARS-CoV-2 viral RNA was extracted from patient's specimen using the PureLink viral RNA/DNA minikit (Invitrogen). After that, the RNA was converted into cDNA by SuperScript VILO cDNA synthesis kit (Invitrogen). The specific regions that had novel mutations were gone under PCR amplification using our previously reported primers [12]. The amplicons were visualized via $1.5 \%$ agarose gel electrophoresis and purified using the PureLink PCR purification kit (Thermo Fisher Scientific, USA). Sanger dideoxy method was implemented to sequence the purified amplicons with $2 \times$ coverage. ABI 3500 and BigDye Terminator version 3.1 cycle sequencing kit (Applied Biosystems, USA) were used for sequencing.

\section{Results}

The overall scheme of the present study has been described in Fig. 1.

\section{Transversion mutations are predominant in SARS-CoV-2} NIB-1

SARS-CoV-2 NIB-1 is a member of the GR clade (Table 1). The genome contains 11 mutations in total. Among them, 6 were transversion mutations, and 5 were transition mutations. According to GISAID, 7 of these mutations were reported previously, and 4 of them were first identified in SARS-CoV-2 NIB-1 isolate. In these 7 mutations, 4 were transition mutations: three Purine $\rightarrow$ Purine transitions (Table 1; Serial \# 4-6) and one Pyrimidine $\rightarrow$ Pyrimidine transition (Table 1; Serial \# 1). The other three mutations were transversion mutations: two Purine $\rightarrow$ Pyrimidine transversions (Table 1; Serial \# 2,3) and one Pyrimidine $\rightarrow$ Purine transversion (Table 1; Serial \# 7). The 4 novel mutations were located in the Orf1b gene. Among them, only A889V substitution occurred due to C $\rightarrow \mathrm{T}$ (Pyrimidine $\rightarrow$ Pyrimidine) transition. The other 3 mutations were transversion substitutions: two Purine $\rightarrow$ Pyrimidine transversions (Table 1; Serial \# 9, 11) and one Pyrimidine $\rightarrow$ Purine transversion.

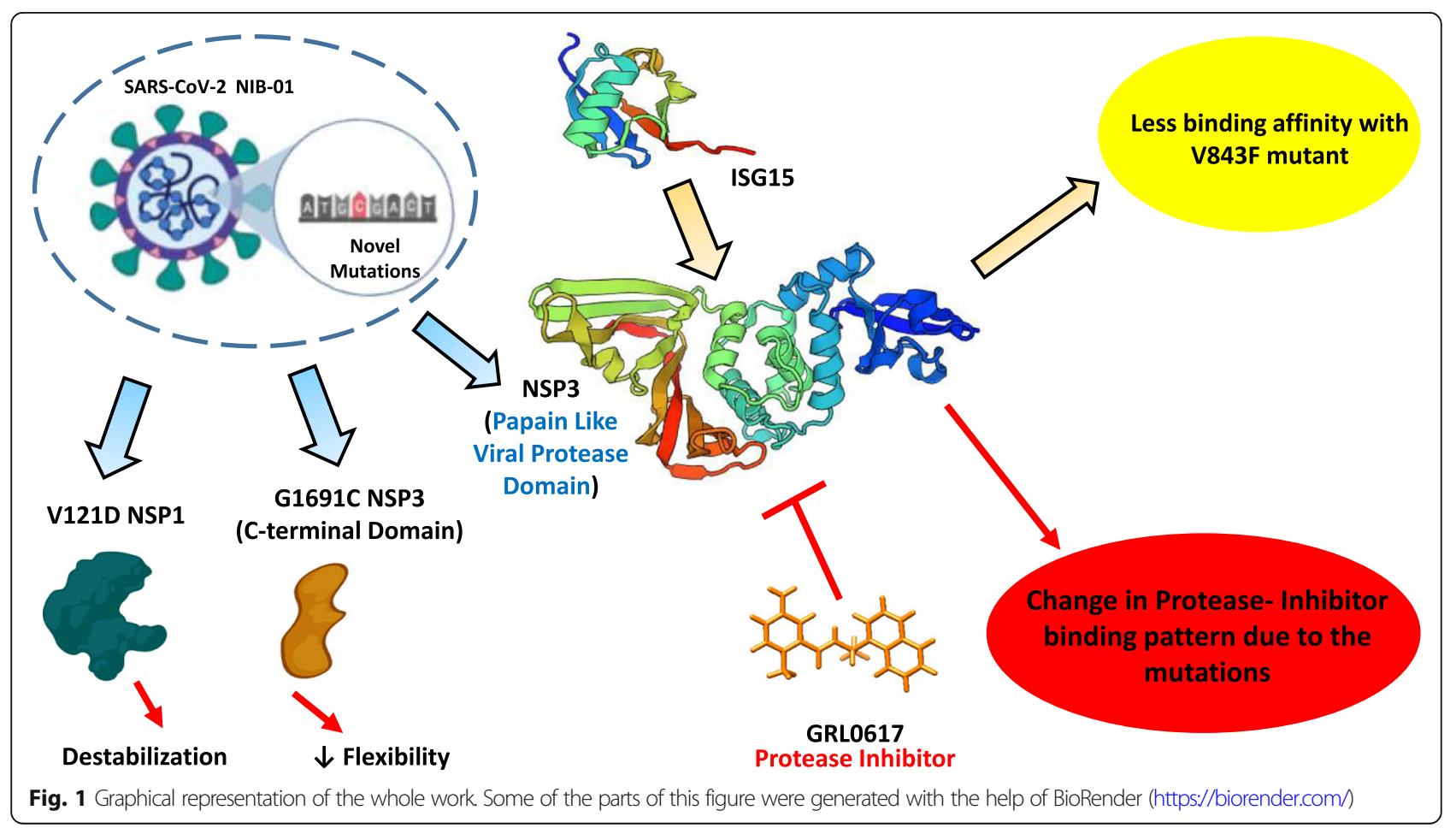


Novel mutations have the potential to alter the functions and structures of protein

According to the MUpro, V121D, V843F, and G1691C can destabilize the proteins (Table 2). Moreover, PROVEAN determined that the V121D and G1691C substitutions are deleterious for the biological functions of the proteins. HOPE demonstrated that most of the mutant amino acids are located in a conserved region, and they are bigger than the wild-type residues. They have the potential to abolish the protein functions. In contrast, only A889V mutant PLPro can increase protein stability (Table 2).

\section{Interaction patterns of GRL0617-PLPro altered in mutant proteases}

The interactions between the inhibitor GRL0617 and PLPro were identified via molecular docking simulation (Fig. 2). Before molecular docking, the quality of the receptors was evaluated by analyzing Ramachandran plots, ERRAT, PROVE, and MolProbity scores. Ramachandran plots showed that the mutant PLPros have 98.1-98.4\% amino acids in favored regions, $1.6-1.9 \%$ in allowed regions, and $0 \%$ in outlier regions. Moreover, the overall quality score of ERRAT was $89.160-91.034 \%$ and mean $\mathrm{Z}$ score was $0.45-0.50$. All of the mutants passed the VERIFY3D evaluations. The MolProbity score of the wild-type crystal structure was 2.55 , while the mutants have MolProbity scores between 1.16 and 1.32 (Supplementary file 3). Therefore, after the homology modeling, the quality of the simulated structures was satisfactory for further analysis $[26,41]$.

V843F PLPro (- $6.7 \mathrm{kcal} / \mathrm{mol})$ and A889V PLPro ($6.9 \mathrm{kcal} / \mathrm{mol}$ ) showed more binding affinity scores than the wild type one $(-6.6 \mathrm{kcal} / \mathrm{mol})$ against GRL0617 (Table 3). However, V843F + A889V PLPro showed slightly less affinity $(-6.2 \mathrm{kcal} / \mathrm{mol})$ than others.

Although the binding affinity did not change significantly due to the mutations, the inhibitor binding site changed along with the interacting amino acids (Table $3)$. The wild-type receptor interacted with ASP164, ARG166, GLU167, SER170, PRO248, and TYR264, whereas ASP76 and ARG82 were common interacting residues for mutant type receptors. Moreover, residues that participated in hydrogen bonding in wild type, single mutants, and double mutants were not the same. V843F and A889V single mutants have the same interacting residue CYS155 which interacted with the inhibitor through hydrogen bonding.

GRL0617 bound mutant PLPros exhibited nearly same root-mean-square deviations (RMSD) value at 50 ns. However, V843F + A889V double mutant exhibited a slightly higher value at the end. The mutations

Table 2 Effect of the novel mutations in protein stability, function, and structure

\begin{tabular}{|c|c|c|c|c|}
\hline $\begin{array}{l}\mathrm{SL} \\
\text { no. }\end{array}$ & $\begin{array}{l}\text { Change of } \\
\text { amino acid }\end{array}$ & $\begin{array}{l}\text { Protein stability } \\
\text { (MUpro) }\end{array}$ & $\begin{array}{l}\text { Protein function (PROVEAN; } \\
\text { cutoff }=-2.5 \text { ) }\end{array}$ & Protein structural properties (HOPE) \\
\hline 1 & V121D & Decrease & Deleterious & $\begin{array}{l}\text { Change of amino acid: } \\
\text { Neutral } \rightarrow \text { Negative } \\
\text { Change in structural stability: } \\
\text { Slight destabilization of NSP-1 } \\
\text { Level of conservation: } \\
\text { Highly conserved }\end{array}$ \\
\hline 2 & V843F & Decrease & Neutral & $\begin{array}{l}\text { Change of amino acid size: } \\
\text { Mutant residue size is bigger } \\
\text { Change in structural stability: } \\
\text { Disturbing the domain and might abolish its function } \\
\text { Level of conservation: } \\
\text { Highly conserved }\end{array}$ \\
\hline 3 & A889V & Increase & Neutral & $\begin{array}{l}\text { Change of amino acid size: } \\
\text { Mutant residue size is bigger. } \\
\text { Change in structural stability: } \\
\text { Disturbing the domain and might abolish its function } \\
\text { Level of conservation: } \\
\text { Located near a highly conserved position, this mutation } \\
\text { might occur without damaging the protein. }\end{array}$ \\
\hline 4 & G1691C & Decrease & Deleterious & $\begin{array}{l}\text { Change of amino acid size: } \\
\text { Mutant residue size is bigger and more hydrophobic. } \\
\text { Change in structural stability: } \\
\text { The wild-type residue is a glycine that might be necessary to } \\
\text { give the protein essential flexibility. Mutation of this glycine } \\
\text { might abolish the function of the protein. } \\
\text { Level of conservation: } \\
\text { The mutant residue is located near a highly conserved position. } \\
\text { The mutant and wild-type residue are not very similar. Based on } \\
\text { this conservation information, this mutation is probably damaging } \\
\text { to the protein. }\end{array}$ \\
\hline
\end{tabular}




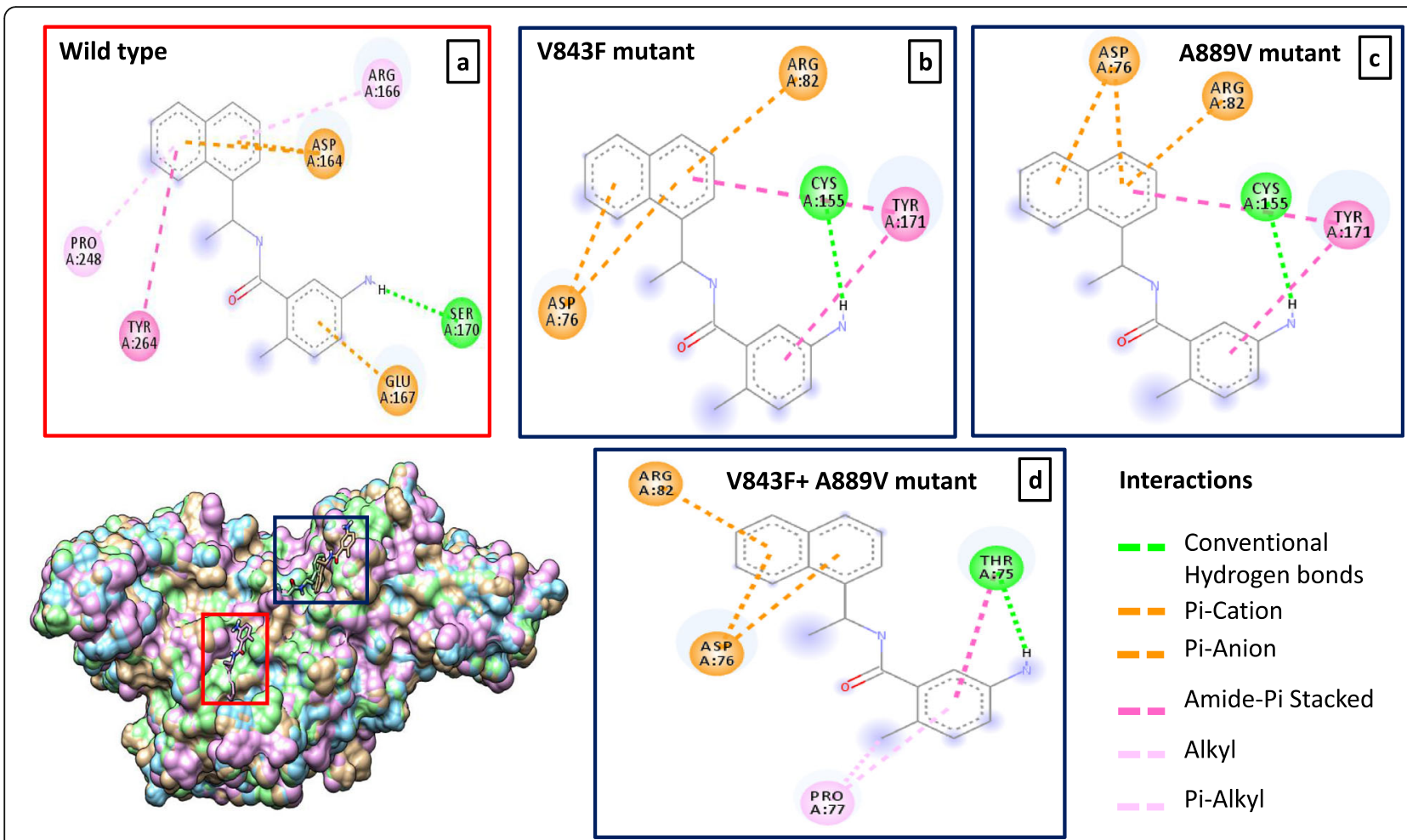

Fig. 2 Interactions between GRL0617 inhibitor and superimposed SARS-CoV-2 wild type and mutant papain-like viral proteases (a-d). Interacting amino acid residues in wild-type and PLPro mutant SARS-CoV-2. Inhibitor binding into the wild type and mutant protein. The red and blue boxes indicate the location and the interactions between ligand and receptors in wild and in mutant proteases, respectively

significantly altered the root-mean-square fluctuations (RMSF) of the amino acid region: 250 to 300. In mutated regions, no remarkable changes of mobility were found. A889V showed a notably altered radius of gyration $(\mathrm{Rg})$ (Supplementary file 5).

\section{V843F PLPro was the most common mutant that} significantly reduced the affinity toward ISG-15

SARS-CoV-2 PLPros cleaves off ISG-15 that is essential to take over the type 1 IFN-mediated antiviral responses [14]. The wild type, A889V and V843F + A889V double mutants showed nearly the same binding affinity $(-15.4$ to $-15.5 \mathrm{kcal} / \mathrm{mol}$ ) toward the $\mathrm{C}$-terminal domain of ISG-15. However, V843F mutant exhibited a significantly lower docking score and binding affinity $(-11.1 \mathrm{kcal} /$ mol) with higher dissociation constant (Table 4). When these mutations were screened in 27 clinical samples, 8 samples $(\sim 29 \%)$ showed V843F mutation in PLPro. The Sanger sequencing reads for both wild type and V843F are given in Supplementary file 4. These V843F-positive patients had mild to moderate symptoms and did not require any intensive care from hospitals.

\section{Discussion}

SARS-CoV-2 is a novel coronavirus and mostly resembles SARS-CoV-1 ( $\sim 80 \%$ sequence similarity). In this study, we focused on SARS-CoV-1-related reports to analyze the results of the mutations [9, 42]. Moreover, we also explored other well-studied betacoronavirus members such as Middle East respiratory syndrome (MERS-CoV), human coronavirus OC43, and mouse

Table 3 Interaction between wild-type and mutant SARS-CoV-2 papain-like viral protease with GRL0617

\begin{tabular}{lll}
\hline Receptor & Binding affinity score $\mathbf{( k c a l / m o l )}$ & Participating amino acids in interaction \\
\hline Wild type & -6.6 & ASP164, ARG166, GLU167, SER170, PRO248, TYR264 \\
V843F & -6.7 & ASP76, ARG82, CYS155, TYR171 \\
A889V & -6.9 & ASP76, ARG82, CYS155, TYR171 \\
V843F + A889V & -6.2 & ASP76, ARG82, THR75, PRO77
\end{tabular}

Italicized residues interacted via hydrogen bonds. 
Table 4 Interaction between wild-type and mutant SARS-CoV-2 papain-like viral protease with ISG-15

\begin{tabular}{lllll}
\hline Receptor & TongDock_A score & Cluster size & Binding affinity score $\mathbf{( k c a l / m o l )}$ & Dissociation constant $\left(\mathbf{K}_{\mathbf{d}}\right)(\mathbf{M})$ \\
\hline Wild type & 915.278 & 41 & -15.4 & $4.8 \mathrm{E}-12$ \\
V843F & 720.354 & 23 & -11.1 & $7.2 \mathrm{E}-09$ \\
A889V & 1019.921 & 31 & -15.4 & $4.7 \mathrm{E}-12$ \\
V843F + A889V & 968.296 & 28 & -15.5 & $4.2 \mathrm{E}-12$ \\
\hline
\end{tabular}

hepatitis coronavirus (MHV) to comprehend different genomic and proteomic functions of SARS-CoV-2.

GISAID CoVsurver revealed that our previously reported isolate 'SARS-CoV-2 NIB-1' is a member of GR clade which mutated 10 times in the coding regions [12]. The NCBI BLASTN unveiled another mutation in the $5^{\prime}$ UTR region. Generally, species are usually biased to transition mutations but in this viral isolate, $54 \%$ of changes occurred due to transversion mutations (Table 1). Transversion mutations are detrimental for RNA viruses (e.g., influenza $\mathrm{A}$ and human immunodeficiency viruses (HIV)) since they can radically change the amino acids $[43,44]$.

The novel mutations V121D, V843F, and G1691C occurred in SARS-CoV-2 NIB-1 isolate due to transversion substitutions that have the potentials to destabilize or alter the protein structure and functions (Table 2). Especially, V121D and V843F positions are highly conserved in the protein, and G1691C might reduce the essential flexibility of NSP-3 (Table 2). Amino acid substitution in V843F and G1691C took place due to $\mathrm{G} \rightarrow \mathrm{T}$ transversion that was possibly introduced by Oxo-guanine generated from reactive oxygen species (ROS) [43-45]. Conversely, $\mathrm{C} \rightarrow \mathrm{T}$ transition, the most frequent transition of SARS-CoV-2, increased the structural stability of PLPro (Table 2) [45]. Besides, the $\mathrm{C} \rightarrow \mathrm{T}$ transition in the $5^{\prime}$ UTR region might interfere with the function of $\mathrm{N}$ and NSP-1 protein [46].

SARS-CoV-2 NIB-1 has V121D and L122I amino acid substitutions in the NSP-1. SARS-CoV-2 NSP-1 has $84.4 \%$ similarity with SARS-CoV-1 NSP-1 [47]. NSP-1 is a potent virulence factor of SARS-CoV-1 that reduces the host mRNA expression by binding with the host $40 \mathrm{~S}$ ribosome to inhibit the translation and specifically accelerate host mRNA degradation keeping the viral mRNA intact $[48,49]$. NSP-1 also disrupts the activation of IFN-dependent antiviral signaling pathways and represses the expression of the innate immune-responding genes such as type I IFN, ISG-56, and ISG-15 [13, 50, 51]. These IFN and ISG-15 gene-mediated antiviral pathways are crucial in host defense mechanisms against SARS-CoV-1 and SARS-CoV-2 [15, 50, 52]. An attenuated SARS-CoV-1 with mutant NSP-1 can replicate as efficiently as wild-type strains with an intact IFN response [52]. Moreover, mutant NSP-1 of MHV exhibited an adequate amount of cytotoxic $\mathrm{T}$ cell generations that protected the mice against further viral infections [53]. Hence, there is a higher possibility that a suitable NSP-1 mutant of SARS-CoV-2 would work as an attenuated vaccine for COVID-19. Through computational analysis, we have observed that V121D and L122I mutants might destabilize the structure of NSP-1 (Table 2 \& Supplementary 2) yet with these two mutations, the virus caused mild fever, cough, and throat congestion in a young female patient [12]. Therefore, V121D and L122I mutations along with 93rd $\mathrm{C} \rightarrow \mathrm{T}$ in $5^{\prime}$ UTR did not change the pathogenicity of SARS-CoV-2 significantly. However, keeping in mind that the quick recovery of the patient within 10 days of the onset of symptoms without any notable care, it might be speculated that NSP-1 with these mutations can be a potent candidate for the investigations and development of an attenuated vaccine against SARS-CoV-2.

Main protease and PLPro are two essential viral enzymes that are vital for polypeptide processing during viral maturation [47]. Therefore, inhibitors of these proteins could yield prospective antiviral drugs [54]. SARSCoV-1 PLPro has been targeted to develop inhibitors for decades [33]. SARS-CoV-1 and SARS-CoV-2 PLPros have 4 domains (Fig. 3) [30]. The active site of this enzyme resides in the thumb domain. PLPro interacts with the $\mathrm{N}$ - and C-terminal domains of ISG-15 through ubiquitin-binding subsites 2 and 1, respectively [33]. This interaction finally cleaves off ISG-15. Hence, type 1 IFN-induced ISG-15 antiviral response cannot function properly in host cells. Consequently, this process hinders innate immune response and enhances viral spread [14]. A study also demonstrated that SARS-CoV-2-infected Vero and Calu3 2B4 cell lines are more susceptible to type 1 INF treatment than SARS-CoV-1 [50, 51]. Most possibly, the IFN treatment increased the synthesis of ISG-15 that ceased the viral replications in the cell lines. PLPro-mediated pathogenesis can be halted with naphthalene-based protease inhibitor GRL0617 since this ligand can make non-covalent bonds with SARSCoV-2 PLPro [14, 30]. Here, V843F and A889V mutations were present in the PLPro domain of the NSP-3 protein. V843F and A889V single mutants and V843F + A889V double mutant changed the binding site of GRL0617. The inhibitor binds in the thumb domain of 


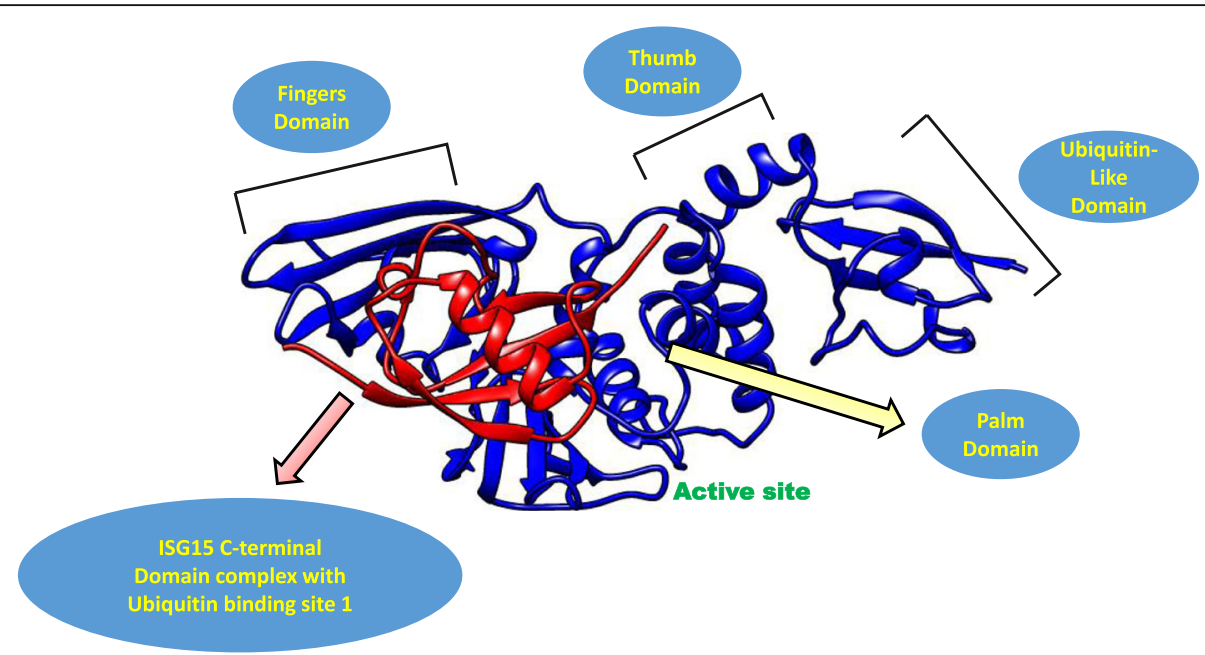

Fig. 3 Interactions between the C-terminal domain of interferon-stimulated gene 15 (ISG-15) and SARS-CoV-2 wild-type papain-like viral proteases. The red chain is the C-terminal domain of ISG-15. The blue chain represents the protease

the wild-type receptor, whereas the mutant receptors interacted through the ubiquitin-like domain (Fig. 2). Although GRL0617-bound mutant PLPros showed similar RMSD at $50 \mathrm{~ns}, \mathrm{~V} 843 \mathrm{~F}+\mathrm{A} 889 \mathrm{~V}$ double mutant demonstrated a slightly higher value at the end. The mutations significantly reduced the mobility of the amino acid region: 250 to 300. According to the PDB ID: 6W9C/ Uniprot ID: P0DTD1, this region has an important role in GRL0617-induced inhibition as mutation in 268th position reduced GRL0617 activity. Additionally, 272th $\mathrm{H}$ is an active site residue. Therefore, these mutations might decrease the efficacy of GRL0617. A889V can change the compactness of the proteins since it showed a notably altered $\mathrm{Rg}$ during the MD simulation [55].There is a chance that these altered binding positions, RMSF, Rg, and RMSD can make the virus resistant against GRL0617. Therefore, GRL0617 may not work well against all variants of SARS-CoV-2.

The mutants of PLPro were also docked against the Cterminal domain of ISG-15. To execute this step, the ISG-15 portion was separated from the PLPro-ISG-15 C-terminal domain complex (PDB ID: 6XA9). Mutant A889V, V843F + A889V, and wild-type PLPro did not show any differences when docked against ISG-15. However, the most frequent mutation V843F led to reduced binding affinity towards the ubiquitin-binding subsite 1 of SARS-CoV-2 PLPro. According to the clinical histories, V843F did not raise pathogenesis in the patients. Therefore, V843F can be assumed as a detrimental and destabilizing transversion mutation that reduced the degradation of ISG-15. Efficient degradation of ISG-15 is a major step to annihilate the innate immune responses. V843F mutation can reduce this process and allow the host cells to halt viral replications. In SARS-CoV-2 NIB1 , A889V substitution protected the protease from the damage of V843F. A889V occurred due to the most common $\mathrm{C} \rightarrow \mathrm{T}$ transition; plausibly this mutation first appeared in the protein, and then V843F took place due to ROS. Yet, further studies are needed to explore how this "yin and yang" incident affects the viral physiology. We hope our findings will give better insights during the development of attenuated vaccines and antiviral drugs.

\section{Conclusion}

This study focused on the effects of novel mutations in SARS-CoV-2 NIB-1 genome. The genome has 4 novel mutations in NSP-1 and NSP-3. These proteins perform essential roles to antagonize IFN responses. A defective or weaker version of these proteins will yield clear insights regarding viral pathogenesis, the pattern of transmission, epidemiology, and therapeutics development.

Our novel mutations will provide helpful insights to create weakened versions of different variants that will emerge in upcoming years. Moreover, our analyses will help to design better universal inhibitors, suitable drugs, or mutant-specific antivirals against PLPro.

\section{Abbreviations \\ SARS-CoV: Severe acute respiratory syndrome coronavirus; PLPro: Papain-like protease; IFN: Interferon; ISG-15: Interferon-stimulating gene-15; \\ PROVEAN: Protein Variation Effect Analyzer; ROS: Reactive oxygen species; PRODIGY: Protein binding energy prediction}

\section{Supplementary Information}

The online version contains supplementary material available at https://doi. org/10.1186/s43141-021-00152-z.

Additional file 1: Supplementary File 1. Parameters for Molecular Docking. The number of Points in Dimension, Spacing (Angstrom) and Center Grid Box values that were taken during the molecular docking of Wild type/ Mutant PLPros and GRL0617. 
Additional file 2: Supplementary File 2. Effect of the Mutations in NSP1 Protein Stability, Function and Structure. MUpro, PROVEAN and HOPE results for mutant L122I NSP1.

Additional file 3: Supplementary File 3. Structural quality reports of Mutant PLPros. The structural quality reports of V843F, A889V and A889V evaluated by RAMPAGE, MolProbity, ERRAT and PROVE.

Additional file 4: Supplementary File 4. Sanger Sequencing Reads for Wild Type and Mutant SARS-CoV-2 PLPro. Sequencing Reads of SARSCoV-2 PLPro V843 coding regions for both Wild type and Mutant isolates. Around 29\% samples showed $\mathbf{G} \rightarrow T$ transversion or V843F substitution.

Additional file 5: Supplementary File 5. Results from Molecular Dynamics (MD) Simulations. RMSD, RMSF and Rg values of GRL0617 bound wild type and mutant PLPro enzymes.

\section{Acknowledgements}

The authors are grateful to the Ministry of Science and Technology, Bangladesh for the extensive support during this work.

\section{Authors' contributions}

MUH, AB, MTHE, ZMC, and ISHA did the data analysis. ISHA, MGM, MM, and MHR helped to write the manuscript. MN, IA, and MRA guided the writing. $A R, K C D, C A K$, and MS conceptualized the study. All of the authors have read and approved the manuscript.

\section{Funding}

There was no funding for this study.

\section{Availability of data and materials}

All of the data are included in the article.

\section{Declarations}

\section{Ethics approval and consent to participate}

Verbal consents were obtained prior to the study. All applicable international, national, and/or institutional guidelines for the care and use of human patients were followed according to the ethics committee of National Institute of Biotechnology (NIB). The ethical approval number is NIB Record No. 2021-01.

\section{Consent for publication}

Not applicable

\section{Competing interests}

The authors declare that they have no competing interests

\section{Author details}

'Bioinformatics Division, National Institute of Biotechnology, Ganakbari, Ashulia, Savar, Dhaka 1349, Bangladesh. ${ }^{2}$ Department of Biochemistry and Microbiology, North South University, Bashundhara, Dhaka 1229, Bangladesh. ${ }^{3}$ Department of Biotechnology and Genetic Engineering, Life Science Faculty, Mawlana Bhashani Science and Technology University, Santosh, Tangail 1902, Bangladesh. ${ }^{4}$ Department of Biochemistry and Molecular Biology, Faculty of Health \& Medical Sciences, Gono Bishwabidyaloy, Ashulia, Savar, Dhaka 1344, Bangladesh. ${ }^{5}$ Molecular Biotechnology Division, National Institute of Biotechnology, Ganakbari, Ashulia, Savar, Dhaka 1349, Bangladesh. ${ }^{6}$ Plant Biotechnology Division, National Institute of Biotechnology, Ganakbari, Ashulia, Savar, Dhaka 1349, Bangladesh. ${ }^{7}$ Center for Medical Biotechnology, MIS, Directorate General of Health Services, Dhaka, Bangladesh. ${ }^{8}$ Department of Microbiology, Mugda Medical College, Dhaka, Bangladesh.

\section{Received: 17 December 2020 Accepted: 22 March 2021} Published online: 02 April 2021

\section{References}

1. Sanche S, Lin Y, Xu C, Romero-Severson E, Hengartner N, Ke R (2020) High contagiousness and rapid spread of severe acute respiratory syndrome coronavirus 2. Emerg Infect Dis 26(7):1470-1477. https://doi.org/10.3201/ eid2607.200282
2. Wu F, Zhao S, Yu B, Chen Y, Wang W, Song Z et al (2020) A new coronavirus associated with human respiratory disease in China. Nature 579(7798):265-269. https://doi.org/10.1038/s41586-020-2008-3

3. Varatharaj A, Thomas N, Ellul M, Davies N, Pollak T, Tenorio E et al (2020) Neurological and neuropsychiatric complications of COVID-19 in 153 patients: a UK-wide surveillance study. Lancet Psychiatry 7(10):875-882. https://doi.org/10.1016/s2215-0366(20)30287-x

4. Bansal M (2020) Cardiovascular disease and COVID-19. Diabetes Metab Syndr Clin Res Rev 14(3):247-250. https://doi.org/10.1016/j.dsx.2020.03.013

5. Verdoni L, Mazza A, Gervasoni A, Martelli L, Ruggeri M, Ciuffreda M et al (2020) An outbreak of severe Kawasaki-like disease at the Italian epicentre of the SARS-CoV-2 epidemic: an observational cohort study. Lancet 395(10239): 1771-1778. https://doi.org/10.1016/s0140-6736(20)31103-x

6. Transmission of SARS-CoV-2: implications for infection prevention precautions. (2020). Retrieved 20 February 2021, from https://www.who.int/ news-room/commentaries/detail/transmission-of-sars-cov-2-implications-forinfection-prevention-precautions

7. Fehr AR, Perlman S (2015) Coronaviruses: an overview of their replication and pathogenesis. Methods Mol Biol 1282:1-23. https://doi.org/10.1007/ 978-1-4939-2438-7__

8. Miłek J, Blicharz-Domańska K (2018) Coronaviruses in avian species - review with focus on epidemiology and diagnosis in wild birds. J Vet Res 62(3): 249-255. https://doi.org/10.2478/jvetres-2018-0035

9. Hossain MU, Bhattacharjee A, Emon M, Chowdhury ZM, Mosaib MG, Mourin M, Das KC, Keya CA, Salimullah M (2021) Recognition of plausible therapeutic agents to combat COVID-19: an Omics data based combined approach. Gene 771:145368. https://doi.org/10.1016/j.gene.2020.145368

10. Khailany R, Safdar M, Ozaslan M (2020) Genomic characterization of a novel SARS-CoV-2. Gene Rep 19:100682. https://doi.org/10.1016/j.genrep.2020.1 00682

11. Shu Y, McCauley J (2017) GISAID: global initiative on sharing all influenza data - from vision to reality. Eurosurveillance 22(13). https://doi.org/10.2 807/1560-7917.es.2017.22.13.30494

12. Moniruzzaman M, Hossain M, Islam M, Rahman M, Ahmed I, Rahman T et al (2020) Coding-complete genome sequence of SARS-CoV-2 isolate from Bangladesh by sanger sequencing. Microbiol Resour Announcements 9(28). https://doi.org/10.1128/mra.00626-20

13. Narayanan K, Ramirez S, Lokugamage K, Makino S (2015) Coronavirus nonstructural protein 1: common and distinct functions in the regulation of host and viral gene expression. Virus Res 202:89-100. https://doi.org/10.101 6/j.virusres.2014.11.019

14. Shin D, Mukherjee R, Grewe D, Bojkova D, Baek K, Bhattacharya A et al (2020) Papain-like protease regulates SARS-CoV-2 viral spread and innate immunity. Nature 587(7835):657-662. https://doi.org/10.1038/s41586-020-2 601-5

15. Morales D, Lenschow D (2013) The antiviral activities of ISG15. J Mol Biol 425(24):4995-5008. https://doi.org/10.1016/j.jmb.2013.09.041

16. Zhang Z, Schwartz S, Wagner L, Miller W (2000) A greedy algorithm for aligning DNA sequences. J Comput Biol 7(1-2):203-214. https://doi.org/10.1 089/10665270050081478

17. Fleischmann WR Jr (1996) Viral genetics. In: Baron S (ed) Medical microbiology, 4th edn. University of Texas Medical Branch at Galveston, Galveston Chapter 43. Available from: https://www.ncbi.nlm.nih.gov/books/ NBK8439/

18. Zisanur Rahman ASM, Bhattacharjee A, Jaber AA, Hossain M, Hasan KN, Islam S, Bhuyan ZA (2020) High-risk non-synonymous SNPs of human Bcl-2 gene alters structural stability and small molecule binding. Biores Commun(BRC) 6(1):791-800 Retrieved from http://bioresearchcommunications.com/ index.php/brc/article/view/20

19. Cheng J, Randall A, Baldi P (2005) Prediction of protein stability changes for single-site mutations using support vector machines. Proteins Struct Funct Bioinform 62(4):1125-1132. https://doi.org/10.1002/prot.20810

20. Choi Y, Chan A (2015) PROVEAN web server: a tool to predict the functional effect of amino acid substitutions and indels. Bioinformatics 31(16):27452747. https://doi.org/10.1093/bioinformatics/btv195

21. Venselaar $H$, te Beek T, Kuipers R, Hekkelman M, Vriend G (2010) Protein structure analysis of mutations causing inheritable diseases. An e-science approach with life scientist friendly interfaces. BMC Bioinformatics 11(1). https://doi.org/10.1186/1471-2105-11-548

22. Berman $\mathrm{H}$ (2000) The protein data bank. Nucleic Acids Res 28(1):235-242. https://doi.org/10.1093/nar/28.1.235 
23. Waterhouse A, Bertoni M, Bienert S, Studer G, Tauriello G, Gumienny R et al (2018) SWISS-MODEL: homology modelling of protein structures and complexes. Nucleic Acids Res 46(W1):W296-W303. https://doi.org/10.1093/ nar/gky427

24. Bhattacharya D, Nowotny J, Cao R, Cheng J (2016) 3Drefine: an interactive web server for efficient protein structure refinement. Nucleic Acids Res 44(W1):W406-W409. https://doi.org/10.1093/nar/gkw336

25. Heo L, Park H, Seok C (2013) GalaxyRefine: protein structure refinement driven by side-chain repacking. Nucleic Acids Res 41(W1):W384-W388. https://doi.org/10.1093/nar/gkt458

26. Lovell S, Davis I, Arendall W, de Bakker P, Word J, Prisant M et al (2003) Structure validation by $C a$ geometry: $\varphi, \psi$ and $C \beta$ deviation. Proteins Struct Funct Bioinform 50(3):437-450. https://doi.org/10.1002/prot.10286

27. Colovos C, Yeates TO (1993) Verification of protein structures: patterns of nonbonded atomic interactions. Protein Sci 2(9):1511-1519. https://doi.org/1 0.1002/pro.5560020916

28. Lüthy R, Bowie JU, Eisenberg D (1992) Assessment of protein models with three-dimensional profiles. Nature 356(6364):83-85. https://doi.org/10.1038/3 $56083 a 0$

29. Pontius J, Richelle J, Wodak SJ (1996) Deviations from standard atomic volumes as a quality measure for protein crystal structures. J Mol Biol 264(1): 121-136. https://doi.org/10.1006/jmbi.1996.0628

30. Freitas B, Durie I, Murray J, Longo J, Miller H, Crich D et al (2020) Characterization and noncovalent inhibition of the deubiquitinase and deISGylase activity of SARS-CoV-2 papain-like protease. ACS Infect Dis 6(8): 2099-2109. https://doi.org/10.1021/acsinfecdis.0c00168

31. Trott O, Olson AJ (2010) AutoDock Vina: improving the speed and accuracy of docking with a new scoring function, efficient optimization, and multithreading. J Comput Chem 31(2):455-461. https://doi.org/10.1002/ jcc. 21334

32. Bhattacharjee A, Hossain M, Chowdhury Z, Rahman S, Bhuyan Z, Salimullah M, Keya C (2020) Insight of druggable cannabinoids against estrogen receptor $\beta$ in breast cancer. J Biomol Struct Dyn 39(5):1-10. https://doi.org/1 0.1080/07391102.2020.1737233

33. Báez-Santos Y, St. John S, Mesecar A (2015) The SARS-coronavirus papain-like protease: structure, function and inhibition by designed antiviral compounds. Antivir Res 115:21-38. https://doi.org/10.1016/j.antiviral.2014.12.015

34. Pettersen E, Goddard T, Huang C, Couch G, Greenblatt D, Meng E, Ferrin T (2004) UCSF chimera? A visualization system for exploratory research and analysis. J Comput Chem 25(13):1605-1612. https://doi.org/10.1002/jcc.20084

35. Abraham M, Murtola T, Schulz R, Páll S, Smith J, Hess B, Lindahl E (2015) GROMACS: high performance molecular simulations through multi-level parallelism from laptops to supercomputers. Softwarex 1-2:19-25. https:// doi.org/10.1016/.j.softx.2015.06.001

36. Schuler L, Daura X, van Gunsteren W (2001) An improved GROMOS96 force field for aliphatic hydrocarbons in the condensed phase. J Comput Chem 22(11):1205-1218. https://doi.org/10.1002/jcc.1078

37. Schüttelkopf AW, van Aalten DM (2004) PRODRG: a tool for highthroughput crystallography of protein-ligand complexes. Acta crystallographica. Sect D Biol Crystallogr 60(Pt 8):1355-1363. https://doi. org/10.1107/S0907444904011679

38. Berendsen H, Grigera J, Straatsma T (1987) The missing term in effective pair potentials. J Phys Chem 91(24):6269-6271. https://doi.org/10.1021/j100308a038

39. Park T, Baek M, Lee H, Seok C (2019) GalaxyTongDock: symmetric and asymmetric ab initio protein-protein docking web server with improved energy parameters. J Comput Chem 40(27):2413-2417. https://doi.org/10.1 002/jcc.25874

40. Xue L, Rodrigues J, Kastritis P, Bonvin A, Vangone A (2016) PRODIGY: a web server for predicting the binding affinity of protein-protein complexes. Bioinformatics:btw514. https://doi.org/10.1093/bioinformatics/btw514

41. Chen V, Arendall W, Headd J, Keedy D, Immormino R, Kapral G et al (2009) MolProbity: all-atom structure validation for macromolecular crystallography. Acta Crystallogr D Biol Crystallogr 66(1):12-21. https://doi.org/10.1107/ s0907444909042073

42. Andersen K, Rambaut A, Lipkin W, Holmes E, Garry R (2020) The proximal origin of SARS-CoV-2. Nat Med 26(4):450-452. https://doi.org/10.1038/s41 591-020-0820-9

43. Gojobori T, Li W, Graur D (1982) Patterns of nucleotide substitution in pseudogenes and functional genes. J Mol Evol 18(5):360-369. https://doi. org/10.1007/bf01733904
44. Lyons DM, Lauring AS (2017) Evidence for the selective basis of transitionto-transversion substitution bias in two RNA viruses. Mol Biol Evol 34(12): 3205-3215. https://doi.org/10.1093/molbev/msx251

45. Koyama, T., Platt, D., \& Parida, L. (2020). Variant analysis of SARS-CoV-2 genomes. Retrieved 20 February 2021, from https://www.who.int/bulletin/ volumes/98/7/20-253591/en/

46. Yang D, Leibowitz $J$ (2015) The structure and functions of coronavirus genomic 3' and 5' ends. Virus Res 206:120-133. https://doi.org/10.1016/j. virusres.2015.02.025

47. Yoshimoto FK (2020) The proteins of severe acute respiratory syndrome coronavirus-2 (SARS CoV-2 or n-COV19), the cause of COVID-19. Protein J 39(3):198-216. https://doi.org/10.1007/s10930-020-09901-4

48. Huang C, Lokugamage K, Rozovics J, Narayanan K, Semler B, Makino S (2011) SARS coronavirus nsp1 protein induces template-dependent endonucleolytic cleavage of mRNAs: viral mRNAs are resistant to nsp1induced RNA cleavage. PLoS Pathog 7(12):e1002433. https://doi.org/10.13 71/journal.ppat.1002433

49. Kamitani W, Narayanan K, Huang C, Lokugamage K, Ikegami T, Ito N, Kubo H, Makino S (2006) Severe acute respiratory syndrome coronavirus nsp1 protein suppresses host gene expression by promoting host mRNA degradation. Proc Natl Acad Sci U S A 103(34):12885-12890. https://doi. org/10.1073/pnas.0603144103

50. Mantlo E, Bukreyeva N, Maruyama J, Paessler S, Huang C (2020) Antiviral activities of type I interferons to SARS-CoV-2 infection. Antivir Res 179: 104811. https://doi.org/10.1016/j.antiviral.2020.104811

51. Lokugamage KG, Hage $A$, de Vries $M$, Valero-Jimenez AM, Schindewolf $C$, Dittmann M, Rajsbaum R, Menachery VD (2020) Type I interferon susceptibility distinguishes SARS-CoV-2 from SARS-CoV. J Virol 94(23): e01410-e01420. https://doi.org/10.1128/JVI.01410-20

52. Wathelet MG, Orr M, Frieman MB, Baric RS (2007) Severe acute respiratory syndrome coronavirus evades antiviral signaling: role of nsp1 and rational design of an attenuated strain. J Virol 81(21):11620-11633. https://doi.org/1 0.1128/JVI.00702-07

53. Züst R, Cervantes-Barragán L, Kuri T, Blakqori G, Weber F, Ludewig B, Thiel V (2007) Coronavirus non-structural protein 1 is a major pathogenicity factor: implications for the rational design of coronavirus vaccines. PLoS Pathog 3(8):e109. https://doi.org/10.1371/journal.ppat.0030109

54. Patick AK, Potts KE (1998) Protease inhibitors as antiviral agents. Clin Microbiol Rev 11(4):614-627. https://doi.org/10.1128/CMR.11.4.614

55. Lobanov M, Bogatyreva N, Galzitskaya O (2008) Radius of gyration as an indicator of protein structure compactness. Mol Biol 42(4):623-628. https:// doi.org/10.1134/s0026893308040195

\section{Publisher's Note}

Springer Nature remains neutral with regard to jurisdictional claims in published maps and institutional affiliations.

\section{Submit your manuscript to a SpringerOpen ${ }^{\circ}$ journal and benefit from:}

- Convenient online submission

- Rigorous peer review

- Open access: articles freely available online

High visibility within the field

- Retaining the copyright to your article

Submit your next manuscript at $>$ springeropen.com 